\title{
Manifestações neurológicas da Covid-19: uma revisão de literatura
}

\author{
Neurological manifestations of Covid-19: a literature review \\ Manifestaciones neurológicas de Covid-19: revisión de la literatura
}

Luis Felipe Silveira Mega ORCID: https://orcid.org/0000-0001-5945-5972

Centro Universitário Barão de Mauá, Brasil

E-mail: luisfelipemega@gmail.com

Fernanda Odete Souza Rodrigues ORCID: https://orcid.org/0000-0002-7340-4351

Universidade de Itaúna, Brasil

E-mail: nandasouzarodrigues@hotmail.com

Júlia Silva Contaifer

ORCID: https://orcid.org/0000-0002-5090-445X

Universidade de Brasília, Brasil

E-mail: juliacontaifer@gmail.com

Carlos Alberto Cavalcante Junior

ORCID: https://orcid.org/0000-0002-7501-180X

Centro Universitário Barão de Mauá, Brasil

E-mail: carloscavalc.jr@gmail.com

Laura de Vasconcelos Machado

ORCID: https://orcid.org/0000-0002-8189-0134 Faculdade Ceres, Brasil

E-mail: lauravm251997@gmail.com

Aline Cristina da Silveira

ORCID: https://orcid.org/0000-0002-5203-3766

Centro Universitário Max Plank, Brasil

E-mail: aline.silveira118@al.unieduk.com.br

Isadora Dantas Sakr Khouri

ORCID: https://orcid.org/0000-0002-1598-9750

Universidade Federal de Ouro Preto, Brasil

E-mail: isadoradantas10@hotmail.com

Robertha Muszkat

ORCID: https://orcid.org/0000-0002-2917-1685

Centro Universitário Barão de Mauá, Brasil

E-mail: romuszkat@gmail.com

João Luiz Megale de Campos Lomas

ORCID: https://orcid.org/0000-0002-5342-4902

Universidade de Itaúna, Brasil

E-mail: joaoluizlomasuit@gmail.com

Valnice Machado Portela

ORCID: https://orcid.org/0000-0002-0807-873X

Universidade Nilton Lins, Brasil

E-mail: valniceportela@gmail.com

Ana Paula Sampaio Ferreira Santos Magalhães

ORCID: https://orcid.org/0000-0001-8834-3371

Faculdade Ceres, Brasil

E-mail: dra.anapaulasampaio@hotmail.com

Marylia Skarlatti da Silva Souza

ORCID: https://orcid.org/0000-0002-7534-6669

Faculdade Ceres, Brasil

E-mail: maryliaskarlatti2@hotmail.com

Bianca Marçal Kós

ORCID: https://orcid.org/0000-0001-5790-0817

Universidade Federal do Maranhão, Brasil

E-mail: bianca.mk@discente.ufma.br

Myriam Dantas Pereira

ORCID: https://orcid.org/0000-0002-7775-1027

Universidade de Itaúna, Brasil

E-mail: myriamdantas2014@gmail.com

André Faraco de Andrade Alves

ORCID: https://orcid.org/0000-0001-7375-7061

Universidade Estácio de Sá, Brasil

E-mail: dedefaa@hotmail.com 


\author{
Victória Freitas de Souza Moura \\ ORCID: https://orcid.org/0000-0001-7052-1205 \\ Centro Universitário UniFacid, Brasil \\ E-mail: victoriafreitasdesousamoura@ hotmail.com \\ Tássia Cristina Duarte \\ ORCID: https://orcid.org/0000-0003-4001-8056 \\ Universidade Cidade de São Paulo, Brasil \\ E-mail: tassia_95@hotmail.com
}

\begin{abstract}
Resumo
O presente artigo objetivou analisar as pesquisas publicadas até o momento sobre os sintomas neurológicos da Covid19 e apresentar os principais resultados observados, de modo a propiciar o maior entendimento sobre a ampla dimensão da doença e, assim, contribuir para que a identificação dessa sintomatologia ocorra em tempo hábil. Trata-se de uma revisão bibliográfica do tipo integrativa, norteada por artigos científicos indexados nas bases de dados Google Acadêmico, National Library of Medicine (PubMED), Literatura Latino-Americana e do Caribe em Ciências da Saúde (LILACS) e Scientific Electronic Library Online (SciELO), entre os anos de 2020 e 2021. Para tanto, foram utilizados os descritores "manifestações", "neurológicas", "Covid-19" e seus respectivos correspondentes em inglês e espanhol. Na literatura, faltam conclusões definitivas sobre o tempo de início de manifestações da neuro-Covid, bem como quais são aquelas de maior gravidade. Além disso, o prognóstico desta condição permanece incerto. Sobre a sintomatologia frequentemente relatada, observou-se predomínio de acidente vascular encefálica $(6,0 \%-74,0 \%)$, cefaleia $(9,0 \%-72,0 \%)$, disgeusia $(5,6 \%-41,0 \%)$, anosmia $(5,1 \%-41,0 \%)$, alteração do nível de consciência $(31,0 \%$ - 39,0\%) e convulsão (5,0\% - 29,7\%). Síndrome de Guillain-Barré, meningite e mielopatia foram diagnósticos incomuns. A identificação inicial de disfunções neurológicas é proveniente da anamnese e do exame físico corretamente executados, e, firmado o diagnóstico, o correto manejo do paciente torna-se essencial, sendo que, embora não exista tratamento específico para a sintomatologia neurológica, medidas de suporte e determinadas terapias farmacológicas têm sido adotadas para o cuidado dos enfermos.
\end{abstract}

Palavras-chave: SARS-Cov-2; Manifestações neurológicas; Covid-19.

\begin{abstract}
This article aimed to analyze the research published so far on the neurological symptoms of Covid-19 and present the main results observed, in order to provide a greater understanding of the broad dimension of the disease and, thus, contribute to the identification of this symptomatology occur in a timely manner. This is an integrative literature review, guided by scientific articles indexed in the Google Academic database, National Library of Medicine (PubMED), Latin American and Caribbean Literature in Health Sciences (LILACS) and Scientific Electronic Library Online (SciELO), between the years 2020 and 2021. For this purpose, the descriptors "manifestations", "neurological", "Covid-19" and their respective counterparts in English and Spanish were used. In the literature, definitive conclusions are lacking about the time of onset of neuro-Covid manifestations, as well as which ones are more severe. Furthermore, the prognosis of this condition remains uncertain. Regarding the frequently reported symptoms, there was a predominance of stroke $(6.0 \%-74.0 \%)$, headache $(9.0 \%-72.0 \%)$, dysgeusia $(5.6 \%-41.0 \%)$, anosmia $(5.1 \%-41.0 \%)$, altered level of consciousness $(31.0 \%-39.0 \%)$ and seizure $(5.0 \%-29.7 \%)$. Guillain-Barré syndrome, meningitis, and myelopathy were uncommon diagnoses. The initial identification of neurological disorders comes from correctly performed anamnesis and physical examination, and, once the diagnosis is established, the correct management of the patient becomes essential, and, although there is no specific treatment for neurological symptoms, support measures and certain pharmacological therapies have been adopted for the care of the diseased.
\end{abstract}

Keywords: SARS-CoV-2; Neurologic manifestations; Covid-19.

\title{
Resumen
}

Este artículo tuvo como objetivo analizar la investigación publicada hasta el momento sobre los síntomas neurológicos de Covid-19 y presentar los principales resultados observados, con el fin de brindar una mayor comprensión de la dimensión amplia de la enfermedad y, así, contribuir a la identificación de esta sintomatología. ocurrir de manera oportuna. Se trata de una revisión integradora de la literatura, guiada por artículos científicos indexados en las bases de datos académicas de Google, Biblioteca Nacional de Medicina (PubMED), Literatura Latinoamericana y del Caribe en Ciencias de la Salud (LILACS) y Biblioteca Electrónica Científica en Línea (SciELO), entre los años 2020 y 2021. Para tal efecto, se utilizaron los descriptores "manifestaciones", "neurológico", "Covid-19" y sus respectivas contrapartes en inglés y español. En la literatura no existen derechos definitivos sobre el momento de aparición de las manifestaciones neuro-Covid, así como cuáles son las más graves. Además, el pronóstico de esta afección sigue siendo incierto. En cuanto a los síntomas notificados con frecuencia, hubo un predominio de accidente cerebrovascular $(6,0 \%-74,0 \%)$, dolor de cabeza $(9,0 \%-72,0 \%)$, disgeusia $(5,6 \%-41,0 \%)$ ), anosmia $(5,1 \%-41,0 \%)$, nivel alterado de conciencia $(31,0 \%-39,0 \%)$ y convulsiones $(5,0 \%-29,7 \%)$. El síndrome de Guillain-Barré, la meningitis y la mielopatía fueron diagnósticos poco frecuentes. La identificación inicial de los trastornos neurológicos proviene de la anamnesis y la exploración física correcta, y una vez establecido el diagnóstico, el correcto manejo del paciente se vuelve fundamental, y, aunque no existe un tratamiento específico para los 
síntomas neurológicos, se han adoptado medidas de soporte y determinantes Las terapias farmacológicas. adoptado para el cuidado de los enfermos.

Palabras clave: SARS-CoV-2; Manifestaciones neurológicas; Covid-19.

\section{Introdução}

Em dezembro de 2019, casos de infecção humana pelo vírus SARS-CoV-2 começaram a ser relatados no distrito de Wuhan, na China. Três meses depois, em março de 2020, a pandemia causada pelo novo coronavírus foi declarada pela Organização Mundial da Saúde (OMS), em razão da rápida disseminação geográfica da doença, e, desde então, a afecção tem sido responsável por diversos impactos na vida em sociedade (OMS, 2020a). Além da alta infectividade, a Covid-19 pode levar a desfechos negativos importantes, tendo resultado, conforme dados de dezembro de 2021, em mais de 5 milhões de óbitos em todo o mundo (OMS, 2020b). Outra evolução relevante é a ocorrência de sequelas relacionadas a essa enfermidade, que, embora tenham o sistema respiratório como principal alvo, também podem afetar os aparelhos cardiovascular, gastrointestinal e neurológico (Monte et al., 2020).

No que concerne ao quadro clínico do paciente infectado pelo SARS-CoV- 2, os sintomas típicos são semelhantes aos de um resfriado comum e se apresentam, geralmente, sob a forma de tosse, mialgia e coriza, associados à febre alta e dispneia. Por outro lado, existem manifestações incomuns, consequentes à resposta inflamatória sistêmica individual que pode provocar, em determinados pacientes, abdome agudo em conjunto com diarreia e vômitos (Monte et al., 2021). Ademais, sintomatologia atípica também foi identificada na população pediátrica, como é o caso da grave Síndrome Inflamatória Multissistêmica, e na população geral, que, por vezes, pode intercorrer com o acometimento do sistema neurológico (Brandão et al., 2020; Whittaker et al., 2020).

Sabe-se que inúmeros patógenos conhecidos apresentam neurotropismo, como, por exemplo, os vírus H1N1, Zika, HIV, Herpes e Citomegalovírus, sendo tais agentes responsáveis por acarretar diversos sinais e sintomas relacionados ao sistema nervoso. No quadro neurológico dessas doenças, destacam-se, no caso do Herpes, manifestações como encefalite necrosante aguda, alterações do estado mental, além de crises olfatórias e epilépticas, enquanto na citomegalovirose há relatos de déficits cognitivos, perda auditiva, convulsões e hipotonia. De modo análogo, o SARS-CoV-2 tem chamado atenção da comunidade científica, uma vez que tem sido descrito um comportamento neurotrópico relativamente semelhante ao destas patologias previamente mencionadas (Bem Junior et al., 2020; Nascimento, 2020).

Existem linhas de raciocínio para elucidar os mecanismos de acometimento, por parte do vírus, do sistema nervoso, embora se saiba que a fisiopatologia é complexa e que, por esta razão, não há um consenso global até o momento. Acredita-se que a entrada viral ocorra por meio do receptor da enzima conversora de angiotensina-2 (ECA-2), encontrada também em neurônios, e que, a partir disso, estruturas nervosas sejam devidamente alcançadas. Entre as múltiplas hipóteses já aventadas, podem-se ressaltar as possibilidades de que o vírus seja transferido através das sinapses dos neurônios infectados, de que haja uma invasão da lâmina cribiforme com envolvimento do nervo olfatório ou, ainda, que a rota de disseminação do SARS-CoV2 se dê pela via hematogênica (Zirpe et al., 2020).

Os primeiros relatos sobre o comprometimento neurológico causado pela Covid-19 datam, ainda, do primeiro semestre de 2020 e são oriundos da China, embora esta ação viral também tenha sido detectada na Espanha, Turquia, nos Estados Unidos entre outros países (Pezzini \& Padovani, 2020). Desde então, foram confirmadas uma série de disfunções do sistema nervoso que, a despeito das diferenças na sistematização entre os pesquisadores, englobaram sintomas cujo espectro compreendeu desde quadros leves até àqueles potencialmente fatais (Ellul et al., 2020; Koralnik \& Tyler, 2020). Nesta gama, salientam-se a anosmia e a disgeusia, que se tornaram conhecidas como duas das principais apresentações da Covid-19 e persistem, ainda nos dias de hoje, como formas importantes de reconhecimento da infecção pela população leiga (Ciciarelli $e t$ al., 2020). 
Diante da possibilidade de acometimento neurológico pelo novo coronavírus, é necessário frisar a relevância da atenção básica nesse contexto. Assim, por esta ser a porta de entrada dos usuários para o Sistema de Saúde Único (SUS) e possuir, entre seus atributos, a diretriz de resolutividade e o princípio da integralidade, sua atuação torna-se central (Medina $e t$ al., 2020; Ministério da Saúde, 2020). Dessa maneira, é fundamental que seus profissionais, sobretudo os médicos generalistas, conheçam a possibilidade da sintomatologia neurológica nos pacientes que estão com Covid-19, ou que a tiveram previamente, e, assim, conduzam adequadamente seus casos. Com isso, a atenção básica poderá, de fato, oferecer cuidados oportunos ao doente, evitar referências desnecessárias, minimizar os riscos de sobrecarga dos hospitais e, finalmente, executar plenamente os ideais de resolutividade e integralidade (Sarti et al., 2020; Medina et al., 2020).

Frente a esse cenário e apesar de o comprometimento neurológico ser inegavelmente relevante, estudos definitivamente conclusivos sobre a temática são pouco numerosos. Dito isso e mediante às prováveis repercussões deste no estado de saúde dos pacientes, o presente artigo objetivou analisar as pesquisas publicadas até o momento e apresentar os principais resultados observados, de modo a propiciar o maior entendimento sobre a ampla dimensão da doença e, assim, contribuir para que a identificação dessa sintomatologia como decorrência da Covid-19 ocorra em tempo hábil.

\section{Metodologia}

Trata-se de uma revisão da literatura do tipo integrativa, norteada por artigos científicos publicados nas bases de dados Google Acadêmico, National Library of Medicine (PubMED), Literatura Latino-Americana e do Caribe em Ciências da Saúde (LILACS) e Scientific Electronic Library Online (SciELO), entre os anos de 2020 e 2021. A pesquisa ocorreu entre os dias 10 de dezembro de 2021 e 10 de janeiro do mesmo ano. Para tanto, foram utilizados os descritores "manifestações", "neurológicas", "Covid-19”, bem como seus respectivos correspondentes em inglês e espanhol.

Como critérios de inclusão, apenas textos originais e com dados primários sobre o acometimento neurológico na infecção pelo SARS-CoV-2 foram selecionados. Ao mesmo tempo, artigos de revisão, produções científicas em duplicidade ou, ainda, aquelas que não contemplaram amplamente a temática proposta por este estudo, apesar de possuírem os descritores escolhidos, foram excluídas após a leitura dos resumos. Aplicados os critérios de elegibilidade, a amostra final foi composta por 10 artigos, conforme demonstrado no fluxograma a seguir (Figura 1). Ainda, as principais características dos textos analisados estão indicadas no quadro abaixo (Quadro 1).

Figura 1 - Fluxograma da seleção dos estudos.

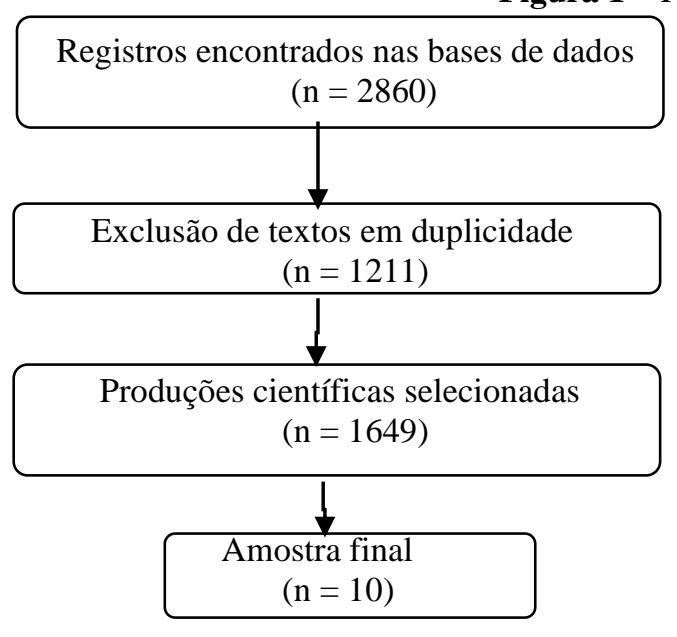

$$
\begin{gathered}
\text { Artigo de revisão e/ou } \\
\text { de } \\
\text { temática incompatível } \\
\qquad(\mathrm{n}=1639)
\end{gathered}
$$

Fonte: Mega et al. (2022). 
Quadro 1 - Especificações dos artigos selecionados e os principais resultados observados pelos autores.

\begin{tabular}{|c|c|c|c|}
\hline ANO & AUTORES & REVISTA & PRINCIPAIS ACHADOS \\
\hline 2020 & $\begin{array}{c}\text { Ebrahimpour et } \\
\text { al. }\end{array}$ & $\begin{array}{c}\text { Caspian Journal of Internal } \\
\text { Medicine }\end{array}$ & $\begin{array}{l}2 \text { pacientes hospitalizados com Covid-19. O primeiro caso apresentou dois episódios } \\
\text { de convulsões tônico-clônicas generalizadas, enquanto a tomografia computadorizada } \\
\text { do cérebro do segundo paciente revelou uma lesão hipodensa extensa no hemisfério } \\
\text { cerebelar esquerdo. }\end{array}$ \\
\hline 2020 & Liotta et al. & $\begin{array}{l}\text { Annals of Clinical and } \\
\text { Translational Neurology }\end{array}$ & $\begin{array}{l}\text { As manifestações neurológicas estavam presentes no início de Covid-19 em } 215 \\
(42,2 \%) \text {, na hospitalização em } 319(62,7 \%) \text { e em qualquer momento durante o curso } \\
\text { da doença em } 419 \text { pacientes }(82,3 \%) \text {. As manifestações neurológicas mais frequentes } \\
\text { foram mialgias }(44,8 \%) \text {, cefaleia }(37,7 \%) \text {, encefalopatia }(31,8 \%) \text {, tontura }(29,7 \%) \text {, } \\
\text { disgeusia }(15,9 \%) \text { e anosmia }(11,4 \%) \text {. Derrames, distúrbios do movimento, déficits } \\
\text { motores e sensoriais, ataxia e convulsões eram incomuns. Fatores de risco } \\
\text { independentes para desenvolver qualquer manifestação neurológica foram Covid-19 } \\
\text { grave e idade mais jovem. }\end{array}$ \\
\hline 2020 & Mao et al. & JAMA Neurology & $\begin{array}{l}\text { Entre os } 214 \text { pacientes hospitalizados com Covid-19, } 78 \text { apresentaram manifestações } \\
\text { neurológicas. Pacientes mais graves eram propensos a ter sintomas neurológicos ( } 40 \\
\text { [45,5\%] versus } 38 \text { [30,2\%]), como doenças cerebrovasculares agudas, consciência } \\
\text { prejudicada e lesão do músculo esquelético. }\end{array}$ \\
\hline 2020 & $\begin{array}{l}\text { Studart-Neto et } \\
\text { al. }\end{array}$ & $\begin{array}{l}\text { Arquivos de Neuro- } \\
\text { Psiquiatria }\end{array}$ & $\begin{array}{l}\text { Foram solicitadas consultas neurológicas para } 89 \text { de } 1.208(7,4 \%) \text { em pacientes } \\
\text { internados por Covid- } 19 \text {. Os principais diagnósticos neurológicos incluíram: } \\
\text { encefalopatia }(44,4 \%) \text {, acidente vascular cerebral }(16,7 \%) \text {, doenças neurológicas } \\
\text { prévias }(9,0 \%) \text {, crises epilépticas }(9,0 \%) \text {, transtornos neuromusculares }(5,6 \%) \text {, outras } \\
\text { lesões encefálicas agudas }(3,4 \%) \text { e outros sintomas leves inespecíficos }(11,2 \%) \text {. }\end{array}$ \\
\hline 2020 & Varatharaj et al. & The Lancet Psychiatry & $\begin{array}{l}77(62,0 \%) \text { de } 125 \text { pacientes apresentaram uma síndrome clínica ampla de um evento } \\
\text { cerebrovascular, dos quais } 57(74,0 \%) \text { tiveram um acidente vascular cerebral } \\
\text { isquêmico e nove }(12,0 \%) \text { uma hemorragia intracerebral. Além dos eventos } \\
\text { cerebrovasculares, } 39(31,0 \%) \text { dos } 125 \text { pacientes apresentaram estado mental alterado, } \\
\text { compreendendo casos de encefalopatia, encefalite e diagnósticos psiquiátricos. } 6 \\
(5,0 \%) \text { apresentaram desordens periféricas, como a síndrome de Guillain-Barré. Os } 3 \\
\text { restantes }(2,0 \%) \text { foram enquadrados em um grupo com outras doenças neurológicas. }\end{array}$ \\
\hline 2021 & Silva et al. & $\begin{array}{c}\text { Revista Brasileira De Terapia } \\
\text { Intensiva }\end{array}$ & $\begin{array}{l}\text { Homem de } 45 \text { anos internado por pneumonia com resultado positivo para SARS-CoV- } \\
2 \text {, sem antecedentes neurológicos, que, ao décimo sexto dia de internação, apresentou } \\
\text { alteração súbita do estado de consciência acompanhada de desvio conjugado do olhar } \\
\text { para a direita e mioclonias da face e da região torácica à esquerda, seguidas de crise } \\
\text { convulsiva tônico-clônica generalizada, associadas à hemiparesia esquerda persistente. } \\
\text { Confirmada existência de RT-PCR positiva para SARS-CoV-2 no líquido } \\
\text { cefalorraquidiano. }\end{array}$ \\
\hline 2021 & $\begin{array}{c}\text { Carcamo Garcia } \\
\text { et al. }\end{array}$ & eNeurologicalSci & $\begin{array}{l}\text { Dos } 199 \text { pacientes com Covid-19 leve a moderado, } 83 \% \text { apresentaram pelo menos } \\
\text { um sintoma neurológico. Com duração média dos sintomas de } 8 \text {. Os sintomas } \\
\text { neurológicos mais comuns foram cefaleia }(72,0 \%) \text {, mialgias }(46,0 \%) \text {, } \\
\text { hipogeusia ou ageusia }(41 \%) \text {, hiposmia ou anosmia }(40,0 \%) \text { e tonturas }(34,0 \%) \text {. A } \\
\text { presença de pelo menos } 1 \text { sintoma neurológico foi associada à presença de algum } \\
\text { sintoma típico da Covid-19, mas não foi relacionada a comorbidades. }\end{array}$ \\
\hline 2021 & $\begin{array}{c}\text { Godoy-Santín et } \\
\text { al. }\end{array}$ & Revista Médica do Chile & $\begin{array}{l}\text { Dos } 1.064 \text { pacientes com Covid-19, } 96 \text { tiveram pelo menos uma nova complicação } \\
\text { neurológica. Os motivos mais comuns de encaminhamento neurológico foram redução } \\
\text { do nível de consciência }(39,0 \%) \text {, déficit neurológico focal }(24,0 \%) \text {, cefaleia }(9,0 \%) \text { e } \\
\text { convulsões }(5,0 \%) \text {. }\end{array}$ \\
\hline 2021 & $\begin{array}{l}\text { Flores-Silva et } \\
\text { al. }\end{array}$ & PLOS One & $\begin{array}{l}\text { Foram estudados } 1.072 \text { casos, } 71 \text { pacientes tinham doenças neurológicas pré- } \\
\text { existentes e } 163(15,2 \%) \text { desenvolveram uma nova complicação neurológica. Dor de } \\
\text { cabeça }(41,7 \%) \text {, mialgia }(38,5 \%) \text {, disgeusia }(8,0 \%) \text { e anosmia }(7,0 \%) \text { foram os } \\
\text { sintomas neurológicos mais comuns na apresentação hospitalar. Delirium }(13,1 \%) \text {, } \\
\text { fraqueza dos membros }(5,1 \%) \text { e recuperação retardada do estado mental após a } \\
\text { retirada da sedação }(2,5 \%) \text { foram as novas síndromes neurológicas mais comuns }\end{array}$ \\
\hline 2021 & Chou et al. & JAMA Network Open & $\begin{array}{l}\text { Entre } 3744 \text { pacientes, as manifestações neurológicas foram encontradas em } \\
\text { aproximadamente } 80,0 \% \text { dos pacientes hospitalizados com Covid-19. Os sintomas } \\
\text { autorreferidos mais comuns incluíram cefaleia }(37,0 \%) \text { e anosmia ou ageusia }(26,0 \%) \text {, } \\
\text { enquanto os sinais neurológicos e/ou síndromes mais comuns foram encefalopatia } \\
\text { aguda }(49,0 \%) \text {, coma }(17,0 \%) \text { e acidente vascular cerebral }(6,0 \%) \text {. A presença de } \\
\text { sinais neurológicos foi associada a um risco aumentado de morte intra-hospitalar. }\end{array}$ \\
\hline
\end{tabular}

Fonte: Mega et al. (2022). 


\section{Resultados}

Este estudo abrangeu uma revisão bibliográfica de dez artigos, os quais foram publicados entre abril de 2020 e julho de 2021, sendo a maior parte composta por trabalhos do tipo coorte. Todas as pesquisas incluídas foram indexadas nas bases de dados supracitadas, não havendo entre elas nenhum ensaio clínico. Do total de sete produções científicas que avaliaram a prevalência de sintomas neurológicos em pacientes com Covid-19, quatro encontraram algum tipo de manifestação em mais de $62,0 \%$ dos pacientes, enquanto as porcentagens de 9,0\%, 15,2\% e 36,0 \% foram identificadas por Godoy-Santín et al., FloresSilva et al. e Mao et al., respectivamente.

No que tange à distribuição de sintomas neurológicos centrais e periféricos, oito trabalhos mencionaram o diagnóstico de ambos os grupos, sendo o primeiro mais frequentemente observado. Os dois artigos restantes, produzidos por Silva et al. e Ebrahimpour et al., constataram apenas sintomas centrais como lesão cerebelar e acidente vascular encefálico (AVE). Tais resultados evidenciaram, portanto, certa uniformidade nas pesquisas selecionadas quanto ao predomínio destes em relação aos periféricos, a exemplo da Síndrome de Guillain-Barré (SGB) e dos déficits motores, como fraqueza muscular, ataxia e outros distúrbios do movimento.

Entre a sintomatologia neurológica mais comum, foi encontrada a cefaleia, cuja incidência oscilou entre 9,0\% e $72,0 \%$, disgeusia $(5,6 \%-41,0 \%)$, alteração do nível de consciência $(31,0 \%-39,0 \%)$, anosmia $(5,1 \%$ - 41,0\%) e convulsão $(5,0 \%$ - 29,7\%). Também se observou que o AVE foi citado por sete autores e sua ocorrência variou entre 6,0\% e 74,0\%. Desordens periféricas como a SGB foram mencionadas apenas por Varatharaj et al. (2020) e Godoy-Santín et al. (2021) e sua incidência esteve entre $1,0 \%$ e 5,0\%, enquanto meningite e mielopatia foram contempladas somente por Chou et al. (2021) e traduziram menos de 1,0\% dos diagnósticos neurológicos identificados pelos autores.

Metade dos textos avaliados não estabeleceu qualquer relação entre os sintomas neurológicos e a evolução do quadro e/ou risco de óbito dos doentes. No entanto, Mao et al. (2021) e Godoy-Santín et al. (2021) demonstraram a existência de uma associação diretamente proporcional entre a ocorrência de encefalopatias e o aumento da mortalidade, ao passo que GodoySantín et al. (2021) relataram que os casos de AVE e de convulsão resultaram em um maior tempo de internação. Paralelamente, Liotta et al. (2020) e Chou et al. (2021) concluíram que qualquer evento neurológico esteve associado a maior gravidade da Covid-19.

Sobre a relação temporal entre o início dos sintomas da Covid-19 e o desenvolvimento de manifestações neurológicas, esta não foi bem definida. Verificou-se que tal surgimento pode ocorrer em qualquer fase do curso da doença, sendo o aparecimento mais tardio abordado por Studart-Neto et al. (2020), que apontaram para o início de sintomas como mialgia persistente e fraqueza após 12 semanas e 6 meses do diagnóstico de infecção pelo SARS-CoV-2, respectivamente. Ainda, a maioria dos textos sugere a possibilidade futura de sequelas neurológicas a curto e longo prazo, embora nenhum deles tenha detectado a presença destas no momento da avaliação das amostras que compuseram as pesquisas.

Com relação à população de maior vulnerabilidade para desenvolver as síndromes neurológicas, nove artigos trataram sobre o assunto e mostraram, como composição, uma maioria formada por homens de 45 a 70 anos e portadores de doenças pré-existentes como hipertensão arterial sistêmica, diabetes mellitus, doença cardíaca ou cerebrovascular e neoplasias. Ao contrário, Carcamo Garcia et al. (2021) demonstraram que a população feminina representou um maior número de atendimentos em determinado centro especializado de neurologia. Quanto às raças e etnias, Liotta et al. (2020) e Chou et al. (2021) notaram maior acometimento entre pessoas brancas, latinas e por aquelas de etnia não hispânica.

\section{Discussão}

A Covid-19 tem se mostrado um grande problema de saúde pública, além de um desafio sem precedentes para a ciência e para a sociedade, cobrando rápidas respostas dos sistemas de saúde diante do surgimento frequente de novas 
descobertas (Medina et al., 2020). A despeito da já mencionada gama de apresentação clínica da doença, com acometimento de órgãos e sistemas que não se restringe ao sistema respiratório, o enfrentamento do novo coronavírus necessita do envolvimento de diversas especialidades médicas. Nessa direção, mais do que infectologistas e pneumologistas, médicos intensivistas, cardiologistas, geriatras e neurologistas, dentre outros, viram suas rotinas modificadas nos últimos dois anos e têm sido cada vez mais desafiados e mobilizados para assistir nas decisões acerca dos cuidados apropriados aos pacientes infectados (Costa et al., 2021).

Desde os primeiros casos de Covid-19 diagnosticados, estudos nacionais e internacionais, dos mais diversos tipos e modalidades, têm sido produzidos e delineados a fim de melhor entender as complexidades deste patógeno, embora muitos deles possam falhar em termos de qualidade (Huth et al., 2021). No que tange ao comprometimento do sistema nervoso no contexto da Covid-19, as pesquisas também são numerosas e seguem em crescimento exponencial na medida em que o conhecimento científico avança diariamente (Mota et al., 2020). No Brasil, percebe-se que há certa restrição quanto à quantidade de publicações originais, sendo estas ainda incipientes, ao passo que a maior parte dos trabalhos divulgados contempla artigos de revisão da literatura (Gomes et al., 2020).

O termo cunhado pelos especialistas para designar o conjunto de sintomas neurológicos causados pelo coronavírus é denominado "neuro-Covid". A denominação desse quadro não é particular ao SARS-CoV-2, descoberta relativamente recente no meio científico. Nesse sentido, outros patógenos que integram a família dos coronavírus, como o SARS-CoV e o MERSCoV, responderam por distúrbios semelhantes e foram responsáveis por epidemias notáveis no Oriente Médio em 2002 e 2012, respectivamente (Prakash et al., 2021). Assim, além da possibilidade de síndrome respiratória aguda grave, foram notificados casos de ataxia, confusão mental, paresia facial, infartos lacunares e encefalite causados por esses agentes (Correia et al., 2020). O maior risco neurológico, todavia, foi observado nos eventos que envolveram o SARS-CoV-2; apesar disso, o conhecimento prévio das outras epidemias pode corroborar para elucidar eventuais dúvidas sobre a Covid-19, uma vez que existem semelhanças entre os vírus mencionados (Verstrepe et al., 2020).

A despeito da não uniformização dos dados sobre a incidência dos quadros neurológicos decorrentes da infecção pelo novo coronavírus, os números evidenciados até então são expressivos. Há estudos que apontaram para a ocorrência dessa sintomatologia em até 90,0\% dos pacientes hospitalizados por Covid-19, enquanto Herman et al. (2020) sinalizaram para uma oscilação de casos entre 6,0\% e 36,0\% durante a doença, sendo esses registros similares aos evidenciados pelos artigos verificados nesta revisão (Puccioni-Sohler et al., 2020). Analogamente ao que foi encontrado nesta pesquisa quanto à distribuição dos sintomas neurológicos entre centrais e periféricos, Mao et al. (2020) observaram que a desordem central foi 3 vezes superior à periférica, enquanto Guerrero et al. (2021) identificaram que a primeira foi $72,6 \%$ mais frequente que a segunda. A observação desses percentuais é de suma importância na rotina médica, já que há certa relação entre os distúrbios centrais e a maior deterioração clínica do doente (Puccioni-Sohler et al., 2020).

A descrição das formas de neuroinvasão que levam às disfunções neurológicas é ampla, complexa e compreende uma série de etapas e eventos celulares cadenciados. Após a entrada do vírus pela ECA-2, os danos neurológicos podem ocorrer pela hipóxia, consequente aos casos graves de Covid-19, pelo ataque autoimune ou pelo aumento da pressão intraluminal (Zirpe et al., 2020; He et al., 2021). Tais mecanismos são relatados em textos variados e figuram como os mais aceitos a esse respeito. Deve-se ressaltar, ainda, que a atuação do SARS-CoV-2 parece não diferir com relação aos sistemas nervosos central e periférico, uma vez que os estudos que retrataram a fisiopatologia viral apresentaram maior enfoque sobre o primeiro, não demonstrando quaisquer diferenças ou especificidades do acometimento periférico (Gaujac \& Amaral, 2021; Silva et al., 2021).

Percebe-se, conforme intensa análise da literatura, que a frequência do registro de cada distúrbio neurológico é variável. Alguns sintomas, no entanto, são recorrentes e demonstrados pela maior parte dos trabalhos, como é o caso da 
cefaleia, hipogeusia e ageusia, anosmia, mialgia, encefalopatia, síndrome confusional e do AVC (Munhoz et al., 2020; Ahmad \& Rathore, 2020). Entre as desordens mais raras, observou-se que menores taxas de incidência recaíram sobre a SGB, a encefalomielite disseminada aguda (EDA) e a encefalopatia necrotizante aguda (ENA), sendo as duas últimas não contempladas nos artigos designados para esta revisão. Ainda, a detecção de comprometimentos como as crises convulsivas e o coma sofreu divergência e houve inconclusão quanto ao fato de estes serem consequências diretas da infecção por Covid-19 (Koralnik \& Tyler, 2020; Divani et al., 2020).

Além disso, o conceito de sintomas neurológicos graves e não graves relacionados à Covid-19 não é claramente definido, sendo encontradas variações entre as publicações disponíveis. No entanto, de modo arbitrário, alguns autores se propuseram a estabelecer o que, para eles, caracterizava quadros mais severos, como foram Godoy-Santín et al. (2021) e Whittaker et al. (2020). Tais autores consideraram e apontaram como manifestações neurológicas graves as convulsões, a encefalite, a alteração da consciência e os acidentes vasculares cerebrais, estes com registros percentuais expressivos. Sobre os últimos, evidenciou-se que, para a maior parte dos eventos constatados, havia certa predisposição, tendo ocorrido, preferencialmente, em indivíduos com hipertensão crônica, diabetes mellitus tipo 2, vasculopatias e cânceres (Godoy-Santín et al., 2021; Whittaker et al., 2020).

Quanto à relação temporal das aparições sintomáticas neurológicas, houve dificuldade para que estas fossem datadas com rigor. Nesse sentido, uma vez que as queixas neurológicas comumente indicam um quadro grave de Covid-19, a utilização de sedoanalgesia, associada à ventilação mecânica, é frequente e pode mascarar o início dos sintomas neurais (Silva et al., 2021). Ellul et al. (2020), todavia, verificaram que as manifestações neurológicas começaram entre 1 e 17 dias após o surgimento da sintomatologia respiratória, com exceção de um homem de 60 anos que apresentou confusão dois dias antes da tosse e febre. Espíndola et al. (2020), por sua vez, demonstraram uma média de 5,9 dias para o aparecimento das primeiras disfunções neurológicas. Além disso, esse mesmo estudo apontou que sintomas específicos como a anormalidades de perfusão do cérebro e a encefalomielite aguda disseminada não tiveram êxito na datação.

Certamente, a discussão acerca da existência de grupos com maior predisposição ao comprometimento neurológico da Covid-19 ainda não está finalizada. Em consonância com os trabalhos avaliados por este texto, outras produções científicas também verificaram que os sintomas do sistema nervoso foram mais prevalentes em pacientes do sexo masculino, brancos, de idade mais avançada e em indivíduos com condições prévias como hipertensão arterial, doença pulmonar obstrutiva crônica e diabetes (Flores-Silva et al., 2021; Luigetti et al., 2020). Alguns fatores checados durante a internação, como plaquetopenia, elevação da creatinina e aumento do D-dímero, também foram associados ao maior risco de acometimento do sistema nervoso. Ademais, as variáveis explicitadas representaram, de modo geral, significativos preditores de mortalidade dos doentes, fato que sinaliza um alerta aos profissionais de saúde para que direcionem maior atenção às populações detentoras de algum desses marcadores (Eskandar et al., 2020).

O prognóstico dos pacientes com Covid-19 e manifestações neurológicas é incerto, pois há disparidades nas repercussões sistêmicas e nos possíveis desfechos do quadro. Como proposto pelo estudo de Liotta et al. (2020), os casos de neuro-Covid envolveram um maior tempo de hospitalização, sendo este, em média, 60,0\% superior ao daquele enfrentado por indivíduos sem essa sintomatologia. Quanto às taxas de mortalidade, estas foram praticamente homogêneas entre pacientes com e sem prejuízos neurofuncionais. No entanto, segundo Beghi et al. (2021), a presença de sintomas neurológicos durante ou após a fase aguda da Covid-19 foi associada a uma doença mais grave e a uma pior evolução, com dados mais expressivos de morte hospitalar (Liotta et al., 2020; Beghi et al., 2021).

A identificação inicial de disfunções neurológicas é proveniente do atendimento médico completo, composto pela anamnese e exame físico corretamente executados, que representa o método padrão para grande parte dos sintomas. Em razão de algumas apresentações clínicas se manifestarem tardiamente, exames complementares são opções viáveis para o diagnóstico 
precoce (Sisniega \& Reynolds, 2021). Nos casos em que há invasão do SNC, a análise do líquor pode ser medida fundamental, devendo ser avaliados parâmetros como o perfil inflamatório, bem como a presença do vírus e de imunoglobulinas como a IgM, sendo a detecção desse anticorpo considerada promissora para firmar o quadro de neuro-Covid (Puccioni-Sohler et al., 2020). Também, a utilização de neuroimagem consiste em ferramenta importante, posto que pode sinalizar anormalidades como micro e macrohemorragias, infartos multifocais, veias hiperdensas, entre outras diversas alterações (Pezzini \& Padovani, 2020).

Feito o diagnóstico, o correto manejo do paciente torna-se essencial. Embora não exista tratamento específico para a sintomatologia neurológica e esta comumente seja autolimitada, medidas de suporte têm sido adotadas, enquanto as farmacológicas podem abranger o uso de imunoglobulinas intravenosas, fatores de crescimento endoteliais antivasculares, imunossupressores e antioxidantes, estes últimos tendo provável efeito neuroprotetor. Além disso, a doença viral pode levar a um estado hipercoagulável que favorece o surgimento de alterações vasculares, como o AVE (Rodriguez et al., 2021; Campos et al., 2020). Nesse aspecto, a avaliação do D-dímero pode ser determinante para o início da terapia anticoagulante, além do reconhecimento de outros fatores de risco, já que níveis elevados desse marcador são correlacionados ao alto risco trombótico. Quando necessária, a anticoagulação dos indivíduos hospitalizados é feita pela heparina, não fracionada ou de baixo peso molecular, com vistas a reduzir os eventos tromboembólicos e melhorar o prognóstico dos enfermos (Campos et al., 2020; Sharma et al., 2021).

Sobre as questões que permanecem em aberto na comunidade científica, deve-se mencionar o fato de a contaminação pelo novo coronavírus poder se associar tanto ao surgimento tardio de doenças neurodegenerativas quanto ao agravamento de condições pré-existentes. A presença prévia dessas enfermidades é capaz, por sua vez, de interferir na sintomatologia da Covid-19, resultando em manifestações clínicas mais severas da afecção viral e piores evoluções do quadro clínico do doente. Dentre as patologias degenerativas comumente relacionadas ao SARS-CoV-2, destacam-se o Parkinson, a demência e a esclerose múltipla, que figuram entre as possíveis consequências diretas da Covid-19. Nessa perspectiva, apesar de os estudos ainda serem inconclusivos, a literatura aponta para prováveis correlações entre a infecção viral e a deterioração nervosa, que pode ser causada pela inflamação neural e/ou pela lesão vascular gerada por esse patógeno, bem como são mencionados, no caso da doença de Parkinson, mecanismos específicos que envolvem alterações na via de síntese da dopamina (Fearon \& Fasano, 2021; McAlpine et al., 2021).

Especulações sobre o tema também são encontradas com relação aos efeitos colaterais das vacinas contra o novo coronavírus, sobretudo quanto ao risco aumentado de eventos adversos neurológicos. Manifestações leves e transitórias como cefaleia, tontura e mialgia são esperadas, enquanto sintomas mais graves são considerados raros. Há casos pontuais de mielite transversa, paralisia de Bell e síndrome de Guillain-Barré, identificada após a aplicação da vacina da Oxford-AstraZeneca como um quadro de neuropatia aguda com fraqueza em membros inferiores; no entanto, não se pode descartar a hipótese de que tais desordens se deram ao acaso (Goss et al., 2021). Sintomatologias como essas podem ser desencadeadas por imunizantes de diversos tipos, inclusive pela vacina contra a gripe, e, por isso, a possibilidade de apresentações clínicas dessa natureza não indica verdadeiros motivos para cessar a campanha de vacinação, uma vez que os benefícios dos imunizantes superam, em muito, os riscos (Garg \& Paliwal, 2021).

Por fim, diante do quadro de neuro-Covid e de seus desdobramentos incertos, vale ressaltar a importância da multidisciplinaridade da equipe de saúde no atendimento ao paciente acometido pela infecção do vírus SARS-CoV-2 e que apresenta disfunções neurológicas. Durante o período de internação, destacam-se os profissionais de enfermagem, farmácia, medicina, fisioterapia, nutrição e psicoterapia na estabilização do quadro clínico dos doentes. O apoio multidisciplinar é um fator de relevância desde o diagnóstico até a evolução e reabilitação desses pacientes, contribuindo para minimizar agravos, possibilitar a recuperação das condições físicas, respiratórias e neurológicas e proporcionar melhor qualidade de vida após a 
alta hospitalar (Fonseca et al., 2021). Ante o exposto, torna-se, também, imprescindível a constante atualização e compreensão das possíveis repercussões neurológicas decorrentes da Covid-19 por todos esses profissionais de saúde, tendo em vista que o fim definitivo do período pandêmico permanece indefinido e que novas variantes têm sido descobertas (Karim \& Karim, 2021; Santos et al., 2021).

\section{Considerações Finais}

Descobertas sobre o novo coronavírus ocorrem constantemente desde os primeiros relatos da infecção humana pelo SARS-CoV-2. Entre elas, as manifestações neurológicas assumem importância significativa dada a sua associação com a maior gravidade do quadro clínico da Covid-19 e ao variado espectro de distúrbios neurofuncionais que foi identificado na literatura. Frente às incertezas sobre o prognóstico do doente, o manejo adequado da neuro-Covid deve envolver desde o conhecimento médico para o diagnóstico precoce dessas condições até as medidas de suporte e/ou farmacológicas, quando estas forem indicadas. A partir desse estudo, espera-se, portanto, que novas pesquisas sejam realizadas a fim de investigar de modo minucioso os diversos aspectos que envolvem esse tema, tais como a relação temporal entre o surgimento dos sintomas neurológicos e as manifestações respiratórias, a evolução da neuro-Covid, bem como os impactos de suas repercussões a longo prazo na qualidade de vida do enfermo.

\section{Referências}

Ahmad, I. \& Rathore, F. A. (2020). Neurological manifestations and complications of COVID-19: A literature review. Journal of clinical neuroscience: official journal of the Neurosurgical Society of Australasia, 77, 8-12.

Beghi, E., Giussani, G., Westenberg, E., Allegri, R., Garcia-Azorin, D., Guekht, A., Frontera, J., Kivipelto, M., Mangialasche, F., Mukaetova-Ladinska, E. B., Prasad, K., Chowdhary, N. \& Winkler, A. S. (2021). Acute and post-acute neurological manifestations of COVID-19: present findings, critical appraisal, and future directions. Journal of Neurology. 21, 1-10.

Bem Junior, L. S., Brito, T. V. B., Ferreira Júnior, A. G., Ribeiro, M. G. L., Alexandrino Júnior, J. S. \& Brito, T. M. M. (2020). Neurotropismo viral: o que já sabemos de semelhanças com o novo coronavírus? COVID-19 no Brasil os múltiplos olhares da ciência para compreensão e formas de enfrentamento, 5 , 6 , 49-59.

Brandão, M. L. L., Rodrigues, F. O. S., Gomes, L. B., Netto, E. L. B., Moura, B. N. G., Lopes, B. C., Menezes, R. V. A., Amaral, L. A., Pereira, B. F. B., Campos, T. F., Aquino, I. P. \& Oliveira, D. L. V. (2021). Síndrome Inflamatória Multissistêmica Pediátrica (SIM-P): Uma análise epidemiológica brasileira. Research, Society and Development, 10, 9, e44710918154.

Campos, L. N., Costa, A. C. S., Cerqueira, D. D. N., Rangel, G. R., Andrade, I. C. F., Souza, S. C. B., Silva, J. C. D. M., Costa, B. M. C. \& Rocha, S. W. S. (2020). COVID-19 neurological manifestations: a narrative review on the mechanisms, pathogenesis, and clinical management. Research, Society and Development, 9, 12, e4291210724.

Carcamo Garcia, M. H., Garcia Choza, D. D., Salazar Linares, B. J. \& Diaz, M. M. (2021). Neurological manifestations of patients with mild-to-moderate COVID-19 attending a public hospital in Lima, Peru. eNeurologicalSci, 23, 100338.

Chou, S. H., Beghi, E., Helbok, R., Moro, E., Sampson, J., Altamirano, V., Mainali, S., Bassetti, C., Suarez, J. I. \& McNett, M. (2021). Global Incidence of Neurological Manifestations Among Patients Hospitalized With COVID-19-A Report for the GCS-NeuroCOVID Consortium and the ENERGY Consortium. JAMA Network Open, 4, 5, e2112131.

Ciciarelli, M. (2020). Academia Brasileira de Neurologia. Manifestações neurológicas do COVID-19. https://www.abneuro.org.br/post/manifesta\%C3\%A7\%C3\%B5es-neurol\%C3\%B3gicas-do-covid-19

Correia, A. O., Feitosa, P., Moreira, J., Nogueira, S., Fonseca, R. B. \& Nobre, M. (2020). Neurological manifestations of COVID-19 and other coronaviruses: A systematic review. Neurology, psychiatry, and brain research, 37, 27-32.

Costa, R., Silva, K. R., Saucedo, S. C. M., Silva, L. A., Crevelari, E. S., Nascimento, W. T. J., Fiorelli, A., Martinelli Filho, M. \& Jatene, F. B. (2021). Impacto da Pandemia por COVID-19 nos Procedimentos Cirúrgicos de Dispositivos Cardíacos Eletrônicos Implantáveis em um Centro de Referência Terciário. Arquivo Brasileiro de Cardiologia, 117, 4, 765-769.

Divani, A. A., Andalib, S., Biller, J., Di Napoli, M., Moghimi, N., Rubinos, C. A., Nobleza, C. O., Sylaja, P. N., Toledano, M., Lattanzi, S., McCullough, L. D., Cruz-Flores, S., Torbey, M. \& Azarpazhooh, M. R. (2020). Central Nervous System Manifestations Associated with COVID-19. Current neurology and neuroscience reports, 20, 12, 60 .

Ebrahimpour, S., Mohseni Afshar, Z., Mohseni, S., Masrour-Roudsari, J., Oladzade, S., Bayani, M. \& Babazadeh, A. (2020). Neurologic manifestations in patients with COVID-19: A case report. Caspian journal of internal medicine, 11, 1, 557-560. 
Ellul, M. A., Benjamin, L., Singh, B., Lant, S., Michael, B. D., Easton, A., Kneen, R., Defres, S., Sejvar, J. \& Solomon, T. (2020). Neurological associations of COVID-19. The Lancet Neurology, 19, 9, 767-783.

Eskandar, E. N., Altschul, D. J., de la Garza Ramos, R., Cezayirli, P., Unda, S. R., Benton, J., Dardick, J., Toma, A., Patel, N., Malaviya, A., Flomenbaum, D., Fernandez-Torres, J., Lu, J., Holland, R., Burchi, E., Zampolin, R., Hsu, K., McClelland, A., Burns, J., Erdfarb, A., \& Mehler, M. F. (2021). Neurologic Syndromes Predict Higher In-Hospital Mortality in COVID-19. Neurology, 96, 11, e1527-e1538.

Espíndola, O. M., Siqueira, M., Soares, C. N., Lima, M. A. S. D., Leite, A. C. C. B., Araujo, A. Q. C., Brandão, C. O. \& Silva, M. T. T. (2020). Patients whit Covid-19 and neurological manifestations show undetectable Sars-Cov-2 RNA levels in the cerebrospinal fluid. International Journal of Infectious Diseases, 96, 567-569.

Fearon, C. \& Fasano, A. (2021). Parkinson's disease and the COVID-19 pandemic. Journal of Parkinson's Disease, 11, 2, $431-444$.

Flores-Silva, F. D., García-Grimshaw, M., Valdés-Ferrer, S. I., Vigueras-Hernández, A. P., Domínguez-Moreno, R., Tristán-Samaniego, D. P., MichelChávez, A., González-Duarte, A., Vega-Boada, F. A., Reyes-Melo, I., Jiménez-Ruiz, A., Chávez-Martínez, O. A., Rebolledo-García, D., Marché-Fernández, O. A., Sánchez-Torres, S., García-Ramos, G., Cantú-Brito, C. \& Chiquete, E. (2021). Neurologic manifestations in hospitalized patients with COVID-19 in Mexico City. PloS one, 16, 4, e0247433.

Fonseca, G. C. D., Silveira, M. F. P., Guimarães, J. Q., Mota, A. L. \& Bezerra, M. A. J. (2021). Atuação da equipe multidisciplinar na reabilitação pósinfecção por SARS-CoV-2. Bionorte, $10,2$.

Garg, R. K. \& Paliwal, V. K. (2021). Spectrum of neurological complications following COVID-19 vaccination. Neurological sciences: official journal of the Italian Neurological Society and of the Italian Society of Clinical Neurophysiology, 1-38.

Gaujac, C. \& Amaral, R. C. (2021). Neurological manifestations and pathophysiological mechanisms of Covid-19. Archives of Health Investigation, 10, 7, 1040-1047.

Godoy-Santín, J., Bravo-Grau, S., Nuñez, F., Aguilar, C., Gutiérrez, D., Miranda, H., Sandoval-Rubio, P., Ramos, B., García A, L., Andresen, M. \& Mellado, P. (2021). Neurología hospitalaria y COVID-19: serie de 96 pacientes evaluados en un hospital universitario. Revista Medica de Chile, 149 , 4, 527-532.

Gomes, A. S., Medeiros Filho, O. B. \& Sousa, M. N. A. (2020). Associação entre o Covid-19 e Manifestações Neurológicas. Brazilian Journal Of Development, 6, 11, 88950-88961.

Goss, A. L., Samudralwar, R. D., Das, R. R. \& Nath, A. (2021). ANA Investigates: Neurological Complications Of Covid-19 Vaccines. Annals Of Neurology, 89, 5, 856-857.

He, Y., Bai, X., Zhu, T., Huang, J., Zhang, H. (2021). What can the neurological manifestations of COVID-19 tell us: a meta-analysis. Journal Of Translational Medicine, 19, 363.

Herman, C., Mayer, K. \& Sarwal, A. (2020). Scoping review of prevalence of neurologic comorbidities in patients hospitalized for COVID-19. Neurology, 95, $2,77-84$

Huth, S. F., Cho, S. M., Robba, C., Highton, D., Battaglini, D., Bellapart, J., Suen, J. Y., Li Bassi, G., Taccone, F. S., Arora, R. C., Whitman, G., Fraser, J. F. \& Fanning, J. P. (2021). Neurological Manifestations of Coronavirus Disease 2019: A Comprehensive Review and Meta-Analysis of the First 6 Months of Pandemic Reporting. Frontiers in neurology, 12, 664599.

Karim, S. \& Karim, Q. A. (2021). Omicron SARS-CoV-2 variant: a new chapter in the COVID-19 pandemic. The Lancet, 398, 10317, 2126-2128.

Koralnik, I. J. \& Tyler, K. L. (2020). COVID-19: A Global Threat to the Nervous System. Annals of Neurology, 88, 1, 1-11.

Liotta, E. M., Batra, A., Clark, J. R., Shlobin, N. A., Hoffman, S. C., Orban, Z. S. \& Koralnik, I. J. (2020). Frequent neurologic manifestations and encephalopathy-associated morbidity in Covid-19 patients. Annals of Clinical and Translational Neurology, 7, 11, 2221-2230.

Luigetti, M., Iorio, R., Bentivoglio, A. R., Tricoli, L., Riso, V., Marotta, J., Piano, C., Primiano, G., Zileri Del Verme, L., Lo Monaco, M. R., Calabresi, P. \& Gemelli Against Covid-19 Group (2020). Assessment of neurological manifestations in hospitalized patients with COVID-19. European journal of neurology, 27, 11, 2322-2328.

Mao, L., Jin, H., Wang, M., Hu, Y., Chen, S., He, Q., Chang, J., Hong, C., Zhou, Y., Wang, D., Miao, X., Li, Y. \& Hu, B. (2020). Neurologic Manifestations of Hospitalized Patients With Coronavirus Disease 2019 in Wuhan, China. JAMA Neurology, 77, 6, 683-690.

McAlpine, L. S., Fesharaki-Zadeh, A. \& Spudich, S. (2021). Coronavirus disease 2019 and neurodegenerative disease: what will the future bring? Current Opinion in Psychiatry, 34, 2, 177-185.

Medina, M. G., Giovanella, L., Bousquat, A., Mendonça, M. H. M. \& Aquino, R. (2020). Atenção primária à saúde em tempos de COVID-19: o que fazer? Caderno de Saúde Pública, 36, 8, e00149720.

Ministério da Saúde (2020). Sistema Único de Saúde (SUS): estrutura, princípios e como funciona, https://www.gov.br/saude/pt-br/assuntos/saude-de-a-a$\mathrm{z} / \mathrm{s} /$ sistema-unico-de-saude-sus-estrutura-principios-e-como-funciona

Monte, L. M., Mendes, L. A., Camargo, R. L., Gomes, R. S. S., Silveira, P. H. A., Seyfarth, M. S. C., Cunha, D. M., Oliveira, L. P. R., Silveira, R. e Silveira, G. R. R. A. (2020). Complicações atípicas e características clínico-epidemiológicas do COVID-19: uma revisão integrativa. Revista Eletrônica Acervo Saúde, 46, e3699.

Mota, D. M., Ferreira, P. J. G. \& Leal, L. F. (2020). Produção científica sobre a COVID-19 no Brasil: uma revisão de escopo. Revista Visa em Debate, 8, 3, 114-124. 
Munhoz, R. P., Pedroso, J. L., Nascimento, F. A., Almeida, S. M., Barsottini, O. G. P., Cardoso, F. E. C. \& Teive, H. A. G. (2020). Neurological complications in patients with SARS-CoV-2 infection: a systematic review. Arquivos de Neuro-Psiquiatria, 78, 5, $290-300$.

Nascimento, O. J. M. (2020). Complicações neurológicas associadas ao SARS-CoV-2 (COVID-19) no Brasil: Organização do grupo NEUROCOVID-RIO e achados preliminares. Revista Brasileira Neurologia, 56, 2, 5-9.

Organização Mundial da Saúde (2020a). WHO Director-General's opening remarks at the midia briefing on COVID-19 - 11 March 2020. https:/www.who.int/director-general/speeches/detail/who-director-general-s-opening-remarks-at-the-media-briefing-on-covid-19---11-march-2020

Organização Mundial da Saúde (2020b). WHO Coronavirus Disease (COVID-19). Dashboard. https://covid19.who.int/

Pezzini, A. \& Padovani, A. (2020). Lifting the mask on neurological manifestations of COVID-19. Nature Reviews Neurology, 16, 636-644.

Prakash, A., Singh, H., Sarma, P., Bhattacharyya, A., Dhibar, D. P., Balaini, N., Shree, R., Goyal, M., Modi, M. \& Medhi, B. (2021). nCoV-2019 infection induced neurological outcome and manifestation, linking its historical ancestor SARS-CoV and MERS-CoV: a systematic review and meta-analysis. Scientific reports, $11,1,12888$.

Puccioni-Sohler, M., Poton, A. R., Franklin, M., Silva, S. J., Brindeiro, R. \& Tanuri, A. (2020). Current evidence of neurological features, diagnosis, and neuropathogenesis associated with COVID-19. Revista da Sociedade Brasileira de Medicina Tropical, 53, e20200477.

Rodríguez, N. C., Bandala, C., Carlo, A. V., Mejía, I. I., Manzo, S. G., Cruz, E. Y. H., Chaverri, J. P., Aparicio, L. C. \& Ochoa, B. H. (2021). Use of antioxidants for the neuro-therapeutic management of COVID-19. Antioxidants (Basel), 10, 6, 971.

Santos, N. N., Ferreira, M. A., Brazil, S. D. S., Cunha, M. S. D., \& Guimarães, B. G. (2021). Sequelas no sistema nervoso central por SARS-CoV-2: uma revisão de literatura. Revista Multidisciplinar em Saúde, 2, 4, 48.

Sarti, T. D., Lazarini, W. S., Fontenelle, L. F. \& Almeida, A. P. S. C. (2020). Qual o papel da Atenção Primária à Saúde diante da pandemia provocada pela COVID-19? Epidemiologia e Serviços de Saúde, 29, 2, e2020166.

Sharma, S., Wood, M. \& Gulli, G. (2021). COVID-19-associated ischaemic stroke despite use of anticoagulation. BMJ Case Reports, 14,7 , e243741.

Silva, F. S. C. A., Bucur, A., Rosado, S. N., Balhana, S. S. \& Meneses-Oliveira, C. M. (2021). Disfunção neurológica associada à COVID-19. Revista Brasileira de Terapia Intensiva, 33, 2, 325.

Sisniega, D. C. \& Reynolds, A. S. (2021). Neurologic Assessment \& Neuromonitoring in the COVID-19 Pandemic. Practical Neurology, 56-60.

Studart-Neto, A., Guedes, B. F., Tuma, R., Camelo Filho, A. E., Kubota, G. T., Iepsen, B. D., Moreira, G. P., Rodrigues, J. C., Ferrari, M., Carra, R. B., Spera, R. R., Oku, M., Terrim, S., Lopes, C., Passos Neto, C., Fiorentino, M. D., DE Souza, J., Baima, J., DA Silva, T., Moreno, C. \& Nitrini, R. (2020). Neurological consultations and diagnoses in a large, dedicated COVID-19 university hospital. Arquivos de neuro-psiquiatria, 78, 8, 494-500.

Varatharaj, A., Thomas, N., Ellul, M. A., Davies, N., Pollak, T. A., Tenorio, E. L., Sultan, M., Easton, A., Breen, G., Zandi, M., Coles, J. P., Manji, H., A1Shahi Salman, R., Menon, D. K., Nicholson, T. R., Benjamin, L. A., Carson, A., Smith, C., Turner, M. R., Solomon, T., \& CoroNerve Study Group (2020). Neurological and neuropsychiatric complications of COVID-19 in 153 patients: a UK-wide surveillance study. The lancet. Psychiatry, 7 , 10, 875-882.

Verstrepen, K., Baisier, L. \& De Cauwer, H. (2020). Neurological manifestations of COVID-19, SARS and MERS. Acta neurologica Belgica, 120, 5, 10511060 .

Whittaker, A., Anson, M., \& Harky, A. (2020). Neurological Manifestations of COVID-19: A systematic review and current update. Acta neurologica Scandinavica, 142, 1, 14-22.

Zirpe, K. G., Dixit, S., Kulkarni, A. P., Sapra, H., Kakkar, G., Gupta, R., Bansal, A. R., Garg, A., Dash, S. K., Gurnani, A., Khan, A., Khatib, K. I. \& Mare, P. R. (2020). Pathophysiological Mechanisms and Neurological Manifestations in COVID-19. Indian journal of critical care medicine: peer-reviewed, official publication of Indian Society of Critical Care Medicine, 24, 10, 975-980. 